\title{
Relation of DNA ploidy to genetic aberrations detected by chromosomal CGH and FISH in gastric adenocarcinomas
}

\author{
TOMOKO FURUYA ${ }^{1}$, TETSUJI UCHIYAMA ${ }^{2}$, ATSUSHI ADACHI ${ }^{2}$, YASUYO CHOCHI ${ }^{1}$, \\ ATSUNORI OGA $^{1}$, SHIGETO KAWAUCHI ${ }^{1}$, KIMIO ISHIGLO ${ }^{3}$ and KOHSUKE SASAKI ${ }^{1}$ \\ ${ }^{1}$ Department of Pathology, Yamaguchi University School of Medicine, Ube $755-8505 ;{ }^{2}$ Department of Surgery, Iwakuni \\ Medical Center, Iwakuni 740-0021; ${ }^{3}$ Department of Surgical Pathology, Shuto General Hospital, Yanai 742-0032, Japan
}

Received December 19, 2005; Accepted February 13, 2006

\begin{abstract}
We analyzed DNA copy number aberrations (DCNAs) by chromosomal comparative genomic hybridization (CGH) in 93 consecutive sporadic gastric adenocarcinomas. In addition, numerical aberrations in chromosomes 7 , 11,17 , and 18 were evaluated by fluorescence in situ hybridization (FISH). Gastric cancers were divided on the basis of nuclear DNA content measured by laser scanning cytometry (LSC) into two groups, 36 DNA diploid (1.0 $\leq$ DNA index $(\mathrm{DI})<1.2$ ) and 57 aneuploid (DI $\geq 1.2$ ) cancers. The most frequent gain and loss of DNA copy number were found at 8q21-23 and 19p13.3, respectively, in both diploid and aneuploid cancers. Diploid cancers were further divided on the basis of genetic aberrations into major type and subtype cancers. The diploid cancer group included nine subtype cancers that showed large numbers of DCNAs; the mean number of DCNAs detected by CGH was 26.7 per tumor. This value was much larger in these diploid subtype cancers than diploid major type cancers (mean, 5.2 per tumor, $\mathrm{p}<0.0001)$. These nine cancers were also characterized by large intercellular variations in chromosome copy numbers that were not detected in the 27 major diploid type cancers. The aneuploid cancer group included only three subtype tumors that showed only a small number of DCNAs (mean, 3 per tumor) and minimal intercellular variations in chromosomal copy number. These data indicate that gastric adenocarcinomas can be divided into three types; aneuploid, major diploid type and diploid subtype cancers. Large-scale studies are necessary to clarify the differences in biological characteristics and underlying genetic mechanisms between these types.
\end{abstract}

Correspondence to: Dr Kohsuke Sasaki, Department of Pathology, Yamaguchi University School of Medicine, Ube 755-8505, Japan

E-mail: kohsuke@yamaguchi-u.ac.jp

Key words: DNA ploidy, genetic instability, fluorescence in situ hybridization, laser scanning cytometry

\section{Introduction}

Cancer is the most common cause of death in advanced countries, and its incidence steadily increases with the elongation of lifespan. Gastric cancer is one of the major causes of cancer deaths in the world. Although early detection coupled with the improvement of diagnostic and therapeutic procedures has increased survival, many patients with gastric cancer still die of the disease. In general, the prognosis of patients with early gastric cancer is excellent and that of patients with advanced gastric cancer is poor. A large variation in the biological behavior of tumors of the same pathological stage has been noted, and the biological characteristics of tumors are considered to be primarily dependent on the genetic alterations of cancer cells. The most effective treatments may be those that are tailored to the individual cancer patient on the basis of the biological characteristics of the tumor. Therefore, tumors should be classified into pathogenetic subtypes with distinct clinical course to design a therapeutic strategy for each cancer patient. To achieve individually-tailored treatments, clinical tools for the diagnosis and classification of patients are needed. Although histological classification is valuable for precise diagnosis of gastric cancers, it allows a limited estimation of the biological characteristics of an individual tumor. DNA ploidy that represents only gross changes in the genome of a cell population is more or less linked with biological characteristics of tumors. Measurements of DNA ploidy are easily implemented and highly costeffective (1-3). Gastric cancers as well as other tumors are divided into two groups, diploid and aneuploid tumors, on the basis of the nuclear DNA content with the intention of optimizing treatment regimens for cancer patients. It is also true, however, that the relationship of DNA ploidy to biological characteristics is tenuous (4-6). This leads to a hypothesis that heterogeneous subtypes within each ploidy group distort the relationship between DNA ploidy and biological characteristics of gastric adenocarcinomas (7). At present, there are few data concerning the existence or nonexistence of subtypes in diploid or aneuploid cancers. If there are subtypes within each group, a new ploidy classification system that takes into account the subtypes must be developed. Since the ploidy status depends on types of genetic instability $(8,9)$, it is a prerequisite to identify subtypes with different genetic characteristics for each ploidy group in 

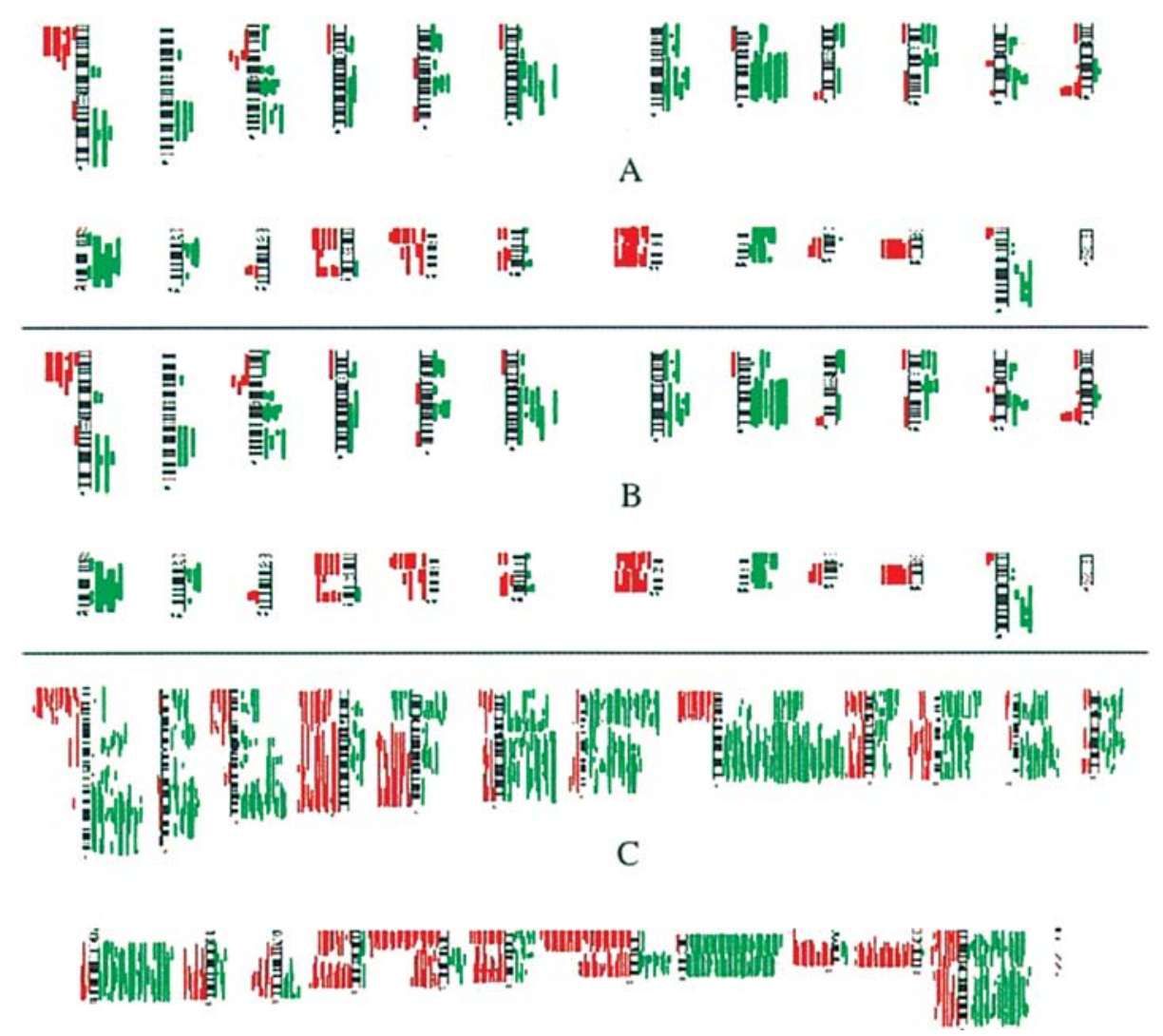

Figure 1. CGH profiles of DNA major diploid type (A), subtype (B) and aneuploid gastric adenocarcinomas (C). Chromosomal regions with DNA copy number gains are shown with green bars to the right of the chromosome ideograms and losses are shown with red bars to the left of the chromosome ideograms. Major diploid cancers $(\mathrm{n}=27)$ are characterized by a small number of DNA copy number aberrations (DNCAs) (mean; 5.2 per tumor, 3.5 gains and 1.8 losses) (A). Subtype cancers $(\mathrm{n}=9)$ are characterized by large number of DNCAs (mean; 26.7 per tumor, 17.1 gains and 9.6 losses). Aneuploid cancers $(\mathrm{n}=57)$ show many DCNAs (mean; 15.2 per tumor, 9.6 gains and 5.6 losses).

primary gastric cancers for adoption of the new classification system.

In the present study, we examined genetic characteristics of gastric adenocarcinomas that could be used to define subtypes of diploid and aneuploid tumors. We propose a new ploidy classification system for gastric adenocarcinomas in the present manuscript. On the basis of genetic alterations, gastric adenocarcinomas can be divided into three groups, aneuploid, major diploid, and diploid subtype cancers.

\section{Materials and methods}

Specimens. We used 93 consecutive, surgically removed gastric cancers comprising of 6 early (6 submucosal tumors) and 87 advanced cancers. The patients consisted of 67 males and 26 females with an average age of 69.9 years ranging from 44 to 88 years. Family histories were noncontributory for all patients, and all tumors were considered to be sporadic. Tumor tissue specimens were taken from a representative part of the tumor and, as a control, an additional specimen was taken from the mucosa distal to the tumor. The tissue specimens were stored at $-80^{\circ} \mathrm{C}$ until use. The study protocol was approved in 2004 by the Institutional Review Board for Human Use at the Yamaguchi University School of Medicine, and informed consent for participation in this study was obtained from every patient.
Touch-smear preparations for fluorescence in situ hybridization (FISH) and laser scanning cytometry (LSC). At least five touch smears were prepared by touching thawed tissue specimens to glass slides after wiping blood from the cut surface of the specimens with a paper towel. One touch-smear slide was fixed in $70 \%$ ethanol for measurement of nuclear DNA content by LSC (10-12). The other slides were air dried and fixed with $100 \%$ ethanol for analysis of numerical chromosomal aberrations by FISH (11-13). In addition, slides were subjected to May-Grunwald Giemsa staining for morphological observation. Tumor samples were not used for this study when slides contained a low percentage $(<30 \%)$ of tumor cells.

DNA ploidy measurement by LSC. DNA ploidy was determined as described previously $(10-12,14,15)$. The slides fixed in $70 \%$ ethanol were dipped in a propidium iodide solution $(25 \mu \mathrm{g} / \mathrm{ml}$ in PBS) containing 0.1\% RNase (Sigma, St. Louis, $\mathrm{MO})$. A cover slip was placed on the slide and sealed with nail polish. DNA content was measured with a laser scanning cytometer (LSC 101, Olympus Co., Tokyo, Japan). Usually, more than 2,000 cells were examined for each sample. DNA histograms were generated, and DNA ploidy was determined. The DNA index (DI) was calculated according to principles (16). In this series, tumors with $1.0 \leq \mathrm{DI}<1.2$ were classified as diploid cases, and were separated from DNA aneuploid tumors ( $\mathrm{DI} \geq 1.2$ ) as described previously (12). 
Table I. Average number of DCNAs detected by CGH for types of gastric adenocarcinoma.

\begin{tabular}{|c|c|c|c|}
\hline & \multicolumn{2}{|c|}{ Diploid tumors } & \multirow{2}{*}{$\begin{array}{l}\text { Aneuploid } \\
\text { tumors } \\
(n=57)\end{array}$} \\
\hline & $\begin{array}{l}\text { Major type } \\
\quad(n=27)\end{array}$ & $\begin{array}{l}\text { Subtype } \\
\qquad(n=9)\end{array}$ & \\
\hline $\begin{array}{l}\text { Total number } \\
\text { of DCNAs }{ }^{\mathrm{a}}\end{array}$ & $5.2 \pm 4.0^{\mathrm{b}}$ & $26.7 \pm 8.8^{c}$ & $15.2 \pm 8.5$ \\
\hline $\begin{array}{l}\text { Average number } \\
\text { of gains }\end{array}$ & 3.5 & 17.1 & 9.6 \\
\hline $\begin{array}{l}\text { Average number } \\
\text { of losses }\end{array}$ & 1.8 & 9.6 & 5.6 \\
\hline
\end{tabular}

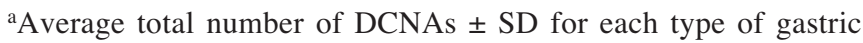
adenocarcinoma. ${ }^{b} \mathrm{p}<0.0001$; major diploid type tumors vs. diploid subtype and aneuploid tumors. ${ }^{c} \mathrm{p}<0.001$; diploid subtype tumors vs. aneuploid type tumors $(\mathrm{p}<0.001)$.

Histological diagnosis. Histological diagnoses were made from routine $5-\mu \mathrm{m}$ sections stained with hematoxylin and eosin according to the Japanese Classification of Gastric Carcinoma (17).

Tissue microdissection and genomic DNA extraction. We used a manual microdissection technique as described previously to reduce the contamination of tumor samples by normal tissue for chromosomal comparative genomic hybridization (CGH) analysis (18). High molecular weight genomic DNA was extracted from each tumor specimen using a DNA extraction kit (SepaGene, Sankojunyaku Co., Ltd, Tokyo, Japan) according to the manufacturer's instructions. Control DNA was extracted from peripheral blood lymphocytes of healthy volunteers.

Chromosomal CGH. Chromosomal CGH and digital image analyses were carried out as described previously (19-23). Briefly, DNAs extracted from tumors and lymphocytes were labeled by nick translation with SpectrumGreen (Vysis, Inc., Downers Grove, IL) and SpectrumRed (Vysis, Inc.), respectively. Labeled DNA samples were co-hybridized onto normal denatured metaphase chromosomes for $72 \mathrm{~h}$ at $37^{\circ} \mathrm{C}$. The slides were then mounted in anti-fade solution containing $0.15 \mathrm{mg} / \mathrm{ml} \mathrm{4,} \mathrm{6-diamino-2-phenylindole} \mathrm{(DAPI)} \mathrm{(Vysis,} \mathrm{Inc.).}$ Images were captured with an Olympus BX60 fluorescence microscope equipped with a $100 \mathrm{X}$ UplanApo objective lens and a cooled charge-coupled device (CCD) camera (SenSys 1400, Photometrics Ltd., Tucson, AZ). Usually, 20 representative images were analyzed for each sample. Increases and decreases in DNA sequence copy number were defined by green-to-red ratios of 1.2 and 0.8 , respectively. The number of DCNAs is represented as the total number of chromosomal regions with alterations in DNA sequence copy number.

FISH. Touch-smear samples fixed in $100 \%$ ethanol were further fixed in $0.2 \%$ paraformaldehyde/PBS at $4^{\circ} \mathrm{C}$ for
10 min as previously described $(11,12)$. We examined numerical aberrations in chromosomes $7,11,17$, and 18 using alphoid satellite DNA probes specific for the pericentromeric region of each chromosome (D7Z1, D11Z1, D17Z1 and D18Z1, respectively; Vysis Inc.) labeled with SpectrumGreen or SpectrumRed (12). FISH was performed according to the manufacturer's instructions as previously described (23). Briefly, $3 \mu 1$ of the probe mixture was applied to ethanol-fixed touch preparations, covered with a coverslip, and then denatured at $73^{\circ} \mathrm{C}$ for $2 \mathrm{~min}$. The slides were incubated overnight at $39^{\circ} \mathrm{C}$ and washed in the washing solution at $45^{\circ} \mathrm{C}$. Then, slides were transferred to a solution of $2 \mathrm{X} \mathrm{SSC} / 0.1 \%$ NP-40 at room temperature. DNA was stained with DAPI-II (Vysis Inc.), and a coverslip was placed on the slide. The number of FISH signals in each nucleus was determined by observing more than 200 nuclei on each slide with an epifluorescence microscope equipped with a $100 \mathrm{X}$ oil immersion objective (Olympus, Tokyo, Japan).

Statistical Analysis. Student's t-test was used to compare two groups of possible permutations of three categories of gastric cancer based on DNA indices. When p-value is $<0.05$ between two groups, statistical difference is considered to be significant.

\section{Results}

Gastric adenocarcinomas were histologically divided into two types, diffuse and intestinal types. In this series, 43 were diffuse type carcinoma and 50 were intestinal type carcinoma. All but six submucosal tumors (invading the submucosa) were advanced tumors. One submucosal carcinoma was classified histologically as diffuse type, and the remaining five were classified as intestinal type.

DNA ploidy analysis by LSC. Of 93 gastric adenocarcinomas, 36 were classified as diploid tumors $(1.0 \leq \mathrm{DI}<1.2)$ and the remaining 57 were classified as aneuploid tumors (DI $\geq 1.2$ ) with DIs ranging from 1.2 to 2.29 . Diffuse type carcinomas tended to be diploid $(25 / 36)$, whereas intestinal type carcinomas tended to be aneuploid (34/57). Four submucosal carcinomas were included in the diploid group, and the remaining two submucosal carcinomas, one of which was diffuse type and the other of which was intestinal type, were classified as aneuploid.

Chromosomal CGH. The number of DCNAs ranged from 0 to 40 in diploid cancers and from 0 to 36 in aneuploid cancers (Fig. 1). In diploid cancers, the average number of DCNAs was $10.8 \pm 10.8$ (mean $\pm \mathrm{SD}$ ), and the average numbers of DNA copy number increases and decreases were 7.3 and 3.7, respectively. In aneuploid cancers, the average number of DCNAs was $15.2 \pm 8.5$ (mean $\pm \mathrm{SD}$ ), and the average numbers of DNA copy number increases and decreases were 9.6 and 5.6, respectively (Table I). Frequently involved chromosomal regions are shown in Table II. The most frequent DNA copy number gain and loss were detected at 8q21-23 and 19p13.3, respectively, in both diploid and aneuploid tumors. Gain of $8 \mathrm{q} 21-23$ was detected in $20(55.6 \%)$ of the 36 diploid tumors and $44(77.2 \%)$ of the 57 aneuploid cancers. Loss of 19p13 was detected in $22(61.1 \%)$ of the diploid and $33(57.9 \%)$ of 
Table II. Chromosomal regions with frequent DNA copy number aberrations detected by CGH in gastric adenocarcinoma.

\begin{tabular}{|c|c|c|c|c|}
\hline \multirow[t]{2}{*}{$\begin{array}{l}\text { Chromosomal } \\
\text { regions }\end{array}$} & \multicolumn{4}{|c|}{$\begin{array}{l}\text { No. of tumors with DNA copy number aberrations } \\
\text { (Total number of tumors; 93) }\end{array}$} \\
\hline & Major type (n=27) & Subtype (n=9) & Aneuploid (n=57) & Statistical analysis \\
\hline -1p32-ter & $7(25.9 \%)$ & $7(77.8 \%)$ & $16(28.1 \%)$ & $\mathrm{a}$ \\
\hline$-4 q 24$ & $0(0 \%)$ & $0(0 \%)$ & $14(24.6 \%)$ & $\mathrm{b}$ \\
\hline$+5 \mathrm{p} 13-14$ & $1(3.7 \%)$ & $5(55.6 \%)$ & $12(21.1 \%)$ & $\mathrm{c}$ \\
\hline$+6 \mathrm{p} 12$ & $1(3.7 \%)$ & $1(11.1 \%)$ & $15(26.3 \%)$ & $\mathrm{b}$ \\
\hline$+6 q 22$ & $1(3.7 \%)$ & $6(66.7 \%)$ & $16(28.1 \%)$ & $\mathrm{c}$ \\
\hline$+7 \mathrm{p} 15-21$ & $1(3.7 \%)$ & $3(33.3 \%)$ & $26(45.6 \%)$ & d \\
\hline$-8 p 22$ & $1(3.7 \%)$ & $1(11.1 \%)$ & $14(24.6 \%)$ & $\mathrm{b}$ \\
\hline$+8 q 21-23$ & $12(44.4 \%)$ & $8(88.9 \%)$ & $44(77.2 \%)$ & d \\
\hline$+11 q 14-22$ & $1(3.7 \%)$ & $5(55.6 \%)$ & $14(24.6 \%)$ & d \\
\hline$-12 q 24$ & $1(3.7 \%)$ & $6(66.7 \%)$ & $2(3.5 \%)$ & $\mathrm{a}$ \\
\hline$+13 q 22$ & $9(33.3 \%)$ & $8(88.9 \%)$ & $26(45.6 \%)$ & $\mathrm{a}$ \\
\hline$-16 \mathrm{p} 13$ & $1(3.7 \%)$ & $6(66.7 \%)$ & $11(19.2 \%)$ & $\mathrm{a}$ \\
\hline$-16 q 23-24$ & $0(0 \%)$ & $6(66.7 \%)$ & $14(24.6 \%)$ & $\mathrm{c}$ \\
\hline$-17 p 12-13$ & $5(7.2 \%)$ & $7(77.8 \%)$ & $26(45.6 \%)$ & d \\
\hline-19 p13 & $14(51.2 \%)$ & $8(88.9 \%)$ & $33(57.9 \%)$ & e \\
\hline$+19 q 12$ & $1(3.7 \%)$ & $0(0 \%)$ & $12(21.1 \%)$ & $\mathrm{b}$ \\
\hline$-19 q 13$ & $8(29.6 \%)$ & $8(88.9 \%)$ & $18(31.6 \%)$ & $\mathrm{a}$ \\
\hline$+20 \mathrm{p} 12$ & $2(7.4 \%)$ & $6(66.7 \%)$ & $36(63.2 \%)$ & d \\
\hline$+20 q 13$ & $4(14.8 \%)$ & $4(44.4 \%)$ & $33(57.9 \%)$ & $\mathrm{b}$ \\
\hline$-21 q 22$ & $2(7.4 \%)$ & $3(33.3 \%)$ & $14(24.6 \%)$ & e \\
\hline$-22 q 13$ & $3(11.1 \%)$ & $7(77.8 \%)$ & $20(35.1 \%)$ & $\mathrm{c}$ \\
\hline
\end{tabular}

${ }^{a} \mathrm{P}<0.05$ for major vs. subtype cancers and subtype vs. aneuploid cancers. ${ }^{b} \mathrm{P}<0.05$ for major vs. aneuploid cancers. ${ }^{\mathrm{c}} \mathrm{P}<0.05$ between groups. ${ }^{\mathrm{d}} \mathrm{P}<0.05$ for major type vs. subtype cancers and major type vs. aneuploid type cancers. ${ }^{\mathrm{e}}$ No statistical significant difference between groups.

the aneuploid cancers. The incidence of DCNAs differed at some chromosomal regions between diploid and aneuploid cancers. DNA copy number gains at $6 \mathrm{p} 12,7 \mathrm{p} 15-21,19 \mathrm{q} 12$, 20p12, and 20q13 and DNA copy number losses at 4q24 and 8 p22 were more frequent in aneuploid than in diploid cancers (Table II).

It is noteworthy that diploid cancers included a subtype in which the number of DCNAs was much greater than in others, which were designated as major diploid cancers (26.7 \pm 8.81 vs. $5.2 \pm 4.0, \mathrm{p}<0.0001$ ) (Table I). In this study, 9 of 36 cancers ( $25 \%$ of diploid carcinomas) were put into the subtype category. The average number of DCNAs was significantly greater in diploid subtype cancers than in aneuploid cancers $(\mathrm{p}<0.001)$ and it was smaller in major diploid type cancers than in aneuploid cancers $(\mathrm{p}<0.0001)$ (Table I). DNA index was 1.0 in seven (78\%) of these subtype cancers, and it was 1.1 and 1.18 in the remaining two. These subtype cancers showed distinct intercellular numerical variation in centromeric signals for centromeric probes (Table III). Diploid subtype cancers were not associated with specific clinicopathological features. In contrast, the aneuploid group contained only three cancers (5.3\% of aneuploid tumors) that showed a small number of DCNAs (mean, 3 per tumor) and minimal intercellular numerical variation in centromeric signals. DIs of these tumors were 1.22, 1.24, and 1.26.
Intercellular variation in the chromosome copy number was detected by FISH. In the normal mucosa, approximately $90 \%$ of cells had two signals for all chromosomes examined, and polysomic ( $>4$ signals) cells were virtually never observed. In diploid cancers except for subtype tumors, disomic cells were predominant for all chromosomes examined (Table III). All diploid subtype cancers showed large intercellular variations in chromosome copy number (Table III). DNA aneuploid tumors (DI $\geq 1.2)$ also exhibited large intercellular variations in chromosome copy number (Table III). The percentage of cells with the modal chromosome number was significantly smaller in both aneuploid and diploid subtype tumors than in major diploid type tumors $(1.0 \leq \mathrm{DI}<1.2)$ $(\mathrm{p}<0.0001)$ (Table III). A new classification of gastric adenocarcinomas based on nuclear DNA content coupled with chromosomal aberrations is shown in Fig. 2.

\section{Discussion}

DNA ploidy analysis has been used to estimate biological features of individual tumors and to divide tumors into diploid and aneuploid groups on the basis of nuclear DNA content. Large amounts of DNA ploidy analysis data have been published for various kinds of cancer, including gastric cancer (1-3). However, the clinical usefulness of ploidy analysis is still 
Table III. The percentage of cells with modal centromeric signal number for chromosomes 7, 11, 17, and 18 in three types of gastric adencarcinoma.

\begin{tabular}{llll}
\hline & \multicolumn{2}{c}{ Diploid tumors } & \multirow{2}{*}{$\begin{array}{c}\text { Aneuploid } \\
\text { tumors }\end{array}$} \\
\cline { 2 - 3 } Chromosome & Major type & Subtype & \\
\hline 7 & $78.2 \pm 12.2 \%$ & $59.2 \pm 18.7 \%$ & $58.7 \pm 17.1 \%$ \\
11 & $84.1 \pm 10.7 \%$ & $58.5 \pm 16.8 \%$ & $63.4 \pm 15.5 \%$ \\
17 & $82.9 \pm 9.4 \%$ & $61.2 \pm 18.1 \%$ & $63.5 \pm 18.9 \%$ \\
18 & $76.9 \pm 12.7 \%$ & $52.3 \pm 8.8 \%$ & $60.1 \pm 17.9 \%$ \\
\hline
\end{tabular}

The percentages in the table indicate mean \pm SD for each chromosome in diploid and aneuploid gastric adenocarcinomas. Statisically significant difference in the average percentage of cells with modal signal number for chromosomes $7,11,17$, and 18 is found between major type and subtype tumors $(\mathrm{p}<0.001)$, and between major type and aneuploid tumors $(\mathrm{p}<0.001)$. However, there is no significant difference between diploid subtype and aneuploid tumors.

controversial (4-6). This suggests that there are subtypes with different genetic characteristics within the same ploidy group. As shown in the present study, we were able to divide diploid gastric adenocarcinomas into two groups, a subtype and a major type on the basis of the number of DCNAs and intercellular variations in chromosome number. Subtype cancers exhibited a greater number of DCNAs and larger intercellular variations in chromosome copy number than major type cancers. Since genetic diploid and aneuploid cancers are genetically distinct, it is possible that aneuploid cancers had contaminated diploid cancers. This possibility can be excluded because seven of nine subtype cancers showed a DI of 1.0. Therefore, subtype cancers can be separated from major diploid cancers. The subtype cancers make up approximately a quarter of diploid cancers in this series. The proportion of subtype cancers is too large to be ignored in gastric adenocarcinomas, and the biological characteristics of subtype cancers need to be clarified through large-scale studies.

Although cells of diploid subtype cancers have diploid DNA content as well as major diploid tumors, subtype tumors concomitantly showed features of chromosomal instability represented by large numbers of DCNAs and large intercellular variations in chromosome copy numbers. In general, diploid and aneuploid cancers are associated with microsatellite instability (MIN) and chromosomal instability (CIN), respectively $(8,9)$. MIN brings subtle changes in nuclear DNA content, while CIN causes distinct alterations in DNA content that are detectable by cytometry $(8,9,11,12)$. The relationship between DNA ploidy and genetic instability is clear between diploid major type and aneuploid cancers. The features of genetic instability may correspond to CIN pattern in diploid subtype cancers. However, the complexity of genetic features of the diploid subtype cancers cannot be explained simply by MIN or CIN. It is suggested that genetic forces other than MIN and CIN may be pertinent to the subtype cancers. Further studies are needed to elucidate the genetic mechanisms that underlie these characteristic changes in diploid subtype cancers.

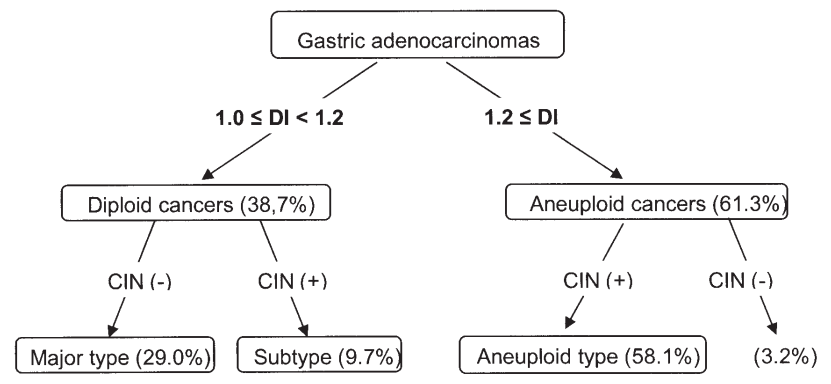

Figure 2. Gastric adenocarcinomas are classified on the basis of nuclear DNA content and genetic abnormalities. First, gastric adenocarcinomas are classified into two types, diploid and aneuploid tumors, and tumors within each type are divided into two types according to the presence or absence of intercellular variation in chromosome copy numbers. Chromosomal instability (CIN) $(+)$ is represented by a large number of DCNAs or large intercellular variations in chromosomal copy number, and CIN (-) is represented by a small number of DCNAs and minimal variations in chromosomal copy number. In this series, $3.2 \%$ of aenuploid cancers do not show CIN features.

In this series, only three $(5.3 \%)$ aneuploid tumors did not show CIN features such as large intercellular variations in chromosome copy number, and their DNA indices were 1.22, 1.24 and 1.26 , which were close to 1.20 . Thus, it is likely that these three tumors were improperly classified as aneuploid. Ploidy classification itself is not difficult, but small errors in DNA content measurement may not be completely excluded. It is practical and appropriate to divide gastric adenocarcinomas into three types: aneuploid, major diploid type and diploid subtype tumors. Because ploidy status together with genetic aberrations is considered to influence the biological characteristics of tumors (25), this new classification system may facilitate personalized treatments of gastric cancer patients. Large-scale studies are necessary to validate the usefulness of the classification system of gastric adenocarcinomas.

\section{Acknowledgements}

We thank Takae Okada and Ai Kihara for their technical help. This study was supported in part by grants from the New Energy and Industrial Technology Development Organization (NEDO) of Japan and the Ministry of Education, Culture, Sports, Science, and Technology of Japan (15659087, 16390107).

\section{References}

1. Abad M, Ciudad J, Rincon MR, et al: DNA aneuploidy by flow cytometry is an independent prognostic factor in gastric cancer. Anal Cell Pathol 16: 223-231, 1998.

2. Baba H, Korenaga D, Kakeji Y, Haraguchi M, Okamura T and Maehara Y: DNA ploidy and its clinical implications in gastric cancer. Surgery 131 (suppl 1): S63-S70, 2004.

3. Dey P: Aneuploidy and malignancy: an unsolved equation. J Clin Pathol 57: 1245-1249, 2004.

4. Lee KH, Lee JS, Lee JH, et al: Prognostic value of DNA flow cytometry in stomach cancer: a 5-year prospective study. $\mathrm{Br} \mathrm{J}$ Cancer 79: 1727-1735, 1999.

5. Sasaki K, Takahashi M, Hashimoto T and Kawachnino K: Flow cytometric DNA measurement of gastric cancers. Clinicopathological implication of DNA ploidy. Pathol Res Pract 184: 561-566, 1989 .

6. Esteban F, De Vega DS, Garcia R, et al: DNA content by flow cytometry in gastric carcinoma: pathology, ploidy and prognosis. Hepatogastroenterology 46: 2039-2043, 1999. 
7. Osterheld MC, Caron L, Demierre M, Laurini R and Bosman FT: DNA-ploidy in advanced gastric carcinoma is less heterogeneous than in early gastric cancer. Cell Oncol 26: 21-29, 2004.

8. Lengauer C, Kinzler KW and Vogelstein B: Genetic instability in colorectal cancers. Nature 386: 623-627, 1997.

9. Lengauer C, Kinzler KW and Vogelstein B: Genetic instabilities in human cancers. Nature 396: 643-649, 1998.

10. Sasaki K, Kurose A, Miura Y, Sato T and Ikeda E: DNA ploidy analysis by laser scanning cytometry (LSC) in colorectal cancers and comparison with flow cytometry. Cytometry 23: 106-109, 1996.

11. Miyazaki M, Furuya T, Shiraki A, Sato T, Oga A and Sasaki K: The relationship of DNA ploidy to chromosomal instability in primary human colorectal cancers. Cancer Res 59: 5283-5285, 1999.

12. Furuya T, Uchiyama T, Murakami T, et al: Relationship between chromosomal instability and intratumoral regional DNA ploidy heterogeneity in primary gastric cancers. Clin Cancer Res 6: 2815-2820, 2000.

13. Sasaki K, Sato T, Kurose A, Uesugi N and Ikeda E: Monosomy of chromosome 18 detected by fluorescence in situ hybridization in colorectal tumors. Cancer 76: 1132-1138, 1995.

14. Kamada T, Sasaki K, Tsuji T, Takahashi M and Kurose A: Sample preparation from paraffin-embedded tissue specimens for laser scanning cytometric DNA analysis. Cytometry 27: 290-294, 1997.

15. Furuya T, Kamada T, Murakami T, Kurose A and Sasaki K: Laser scanning cytometry allows detection of cell death with morphological features of apoptosis in cells stained with PI. Cytometry 29: 1713-1717, 1997.

16. Hiddemann W, Schumann J, Andreeff M, et al: Convention on nomenclature for DNA cytometry. Cytometry 5: 445-446, 1984.

17. Japanese Research Society for Gastric Cancer: Histological findings. In: Japanese Classification of Gastric Carcinoma. 13th edition. Kanehara \& Co., Ltd., Tokyo, pp63-105, 1999.
18. Hashimoto Y, Oga A, Okami K, Imate Y, Yamashita Y and Sasaki K: Relationship between cytogenetic aberrations by CGH coupled with tissue microdissection and DNA ploidy by laser scanning cytometry in head and neck squamous cell carcinoma. Cytometry 40: 161-166, 2000.

19. Suehiro Y, Umayahara K, Ogata H, et al: Genetic aberrations detected by comparative genomic hybridization predict outcome in patients with endometrioid carcinoma. Genes Chromosomes Cancer 29: 75-82, 2000.

20. Oga A, Kong G, Ishii Y, Izumi H, Park CY and Sasaki K: Preferential loss of 5q14-21 in intestinal-type gastric cancer with DNA aneuploidy. Cytometry 46: 57-62, 2001.

21. Kong G, Oga A, Park CK, Kawauchi S, Furuya T and Sasaki K: DNA sequence copy number aberrations associated with histological subtypes and DNA ploidy in gastric carcinoma. Jpn J Cancer Res 92: 740-747, 2001.

22. Harada T, Okita K, Shiraishi K, Kusano N, Kondoh S and Sasaki K: Interglandular cytogenetic heterogeneity detected by comparative genomic hybridization in pancreatic cancer. Cancer Res 62: 835-839, 2002.

23. Ueno T, Tangoku A, Yoshino S, et al: Prediction of nodal metastasis by comparative genomic hybridization in biopsy specimens from patients with superficial esophageal squamous cell carcinoma. Clin Cancer Res 9: 5137-5141, 2003.

24. Ikemoto K, Furuya T, Matsuda K, et al: Multicolor FISH and cytometric analyses allow classification of urothelial carcinomas into two subtypes, low- and high-grade tumors. Int J Oncol 25: 893-898, 2004.

25. Atkin NB: Microsatellite instability. Cytogenet Cell Genet 92: 177-181, 2001. 